Article

\title{
Free Cholesterol Accelerates A $\beta$ Self-Assembly on Membranes at Physiological Concentration
}

\author{
Mohtadin Hashemi ${ }^{1,+}\left(\mathbb{D}\right.$, Siddhartha Banerjee ${ }^{1,2,+}$ and Yuri L. Lyubchenko ${ }^{1, *(D)}$ \\ 1 Department of Pharmaceutical Sciences, University of Nebraska Medical Center, \\ 986025 Nebraska Medical Center, Omaha, NE 68198-6025, USA; mohtadin.hashemi@unmc.edu (M.H.); \\ sbanerjee6@ua.edu (S.B.) \\ 2 Department of Chemistry and Biochemistry, The University of Alabama, Shelby Hall, \\ Tuscaloosa, AL 35487, USA \\ * Correspondence: ylyubchenko@unmc.edu \\ + These authors contributed equally.
}

check for updates

Citation: Hashemi, M.; Banerjee, S.; Lyubchenko, Y.L. Free Cholesterol Accelerates A $\beta$ Self-Assembly on Membranes at Physiological

Concentration. Int. J. Mol. Sci. 2022, 23, 2803. https://doi.org/10.3390/ ijms 23052803

Academic Editor: Andrea Cavalli

Received: 11 February 2022

Accepted: 1 March 2022

Published: 3 March 2022

Publisher's Note: MDPI stays neutral with regard to jurisdictional claims in published maps and institutional affiliations.

Copyright: (C) 2022 by the authors. Licensee MDPI, Basel, Switzerland. This article is an open access article distributed under the terms and conditions of the Creative Commons Attribution (CC BY) license (https:/ / creativecommons.org/licenses/by/ $4.0 /)$

\begin{abstract}
The effects of membranes on the early-stage aggregation of amyloid $\beta(A \beta)$ have come to light as potential mechanisms by which neurotoxic species are formed in Alzheimer's disease. We have shown that direct $A \beta$-membrane interactions dramatically enhance the $A \beta$ aggregation, allowing for oligomer assembly at physiologically low concentrations of the monomer. Membrane composition is also a crucial factor in this process. Our results showed that apart from phospholipids composition, cholesterol in membranes significantly enhances the aggregation kinetics. It has been reported that free cholesterol is present in plaques. Here we report that free cholesterol, along with its presence inside the membrane, further accelerate the aggregation process by producing aggregates more rapidly and of significantly larger sizes. These aggregates, which are formed on the lipid bilayer, are able to dissociate from the surface and accumulate in the bulk solution; the presence of free cholesterol accelerates this dissociation as well. All-atom molecular dynamics simulations show that cholesterol binds $\mathrm{A} \beta$ monomers and significantly changes the conformational sampling of $\mathrm{A} \beta$ monomer; more than doubling the fraction of low-energy conformations compared to those in the absence of cholesterol, which can contribute to the aggregation process. The results indicate that $\mathrm{A} \beta$-lipid interaction is an important factor in the disease prone amyloid assembly process.
\end{abstract}

Keywords: Alzheimer's disease; amyloid aggregation; lipid bilayer; cholesterol; time-lapse AFM imaging; molecular dynamics

\section{Introduction}

The self-assembly of amyloid $\beta(\mathrm{A} \beta)$ is a process that results in the production of neurotoxic oligomer and fibrillar aggregates in Alzheimer's disease [1,2]. Understanding the mechanism by which these aggregates are formed has been the major focus of research in Alzheimer's disease and other fatal neurodegenerative diseases $[3,4]$. However, in the majority of in vitro studies, the $\mathrm{A} \beta$ concentrations used are several orders of magnitude higher than the physiologically relevant concentrations $[5,6]$; no aggregation is observed at the physiological low nanomolar concentration of $A \beta$. This suggests that the aggregation of $A \beta$ in vivo utilizes pathways different from those probed by in vitro experiments.

Recently, an alternative aggregation mechanism has been discovered, allowing for the aggregation to occur at the physiologically relevant concentrations of $A \beta[7,8]$. This is the on-surface aggregation pathway, in which interactions with a surface act as a catalyst for the aggregation process. The model for the on-surface aggregation process suggests that the self-assembly of $A \beta$ oligomers is initiated by the interaction of amyloid proteins with the cellular membrane. The membrane catalyzes amyloid aggregation by stabilizing an aggregation-prone conformation. 
Cell membranes consist of a large variety of lipids, suggesting that numerous factors may contribute to the on-membrane aggregation of amyloids. Indeed, recent publications revealed the role of such lipids as cholesterol (Chol), sphingomyelins, and gangliosides on the formation of $A \beta$ fibrils on membrane surfaces [9-11]. A very recent publication [12] demonstrated that $\mathrm{Chol}$ in the lipid bilayer significantly enhances the aggregation of $\mathrm{A} \beta(1-42)$ at nanomolar monomer concentration. Importantly, computer modeling showed that $A \beta(1-42)$ has an elevated affinity to Chol-containing membranes, adopting a set of aggregation-prone conformations. These studies led to an aggregation model with membranes playing a critical role in triggering the aggregation process and hence, the disease state. Within this model, the membrane composition is a factor controlling the aggregation process, so a change in membrane composition can shift the ratio between monomeric and aggregated states of $A \beta$. This hypothesis is further strengthened by the data regarding the contribution of $\mathrm{Chol}$, sphingomyelins, and gangliosides to the neurotoxicity of $A \beta$ aggregates [13-15], which also highlights these lipids as prime candidates for possible disease defining parameters.

While phospholipids are the major constituent of the cellular lipid bilayer, Chol is the second most abundant lipid and provides stability to the cellular membrane. Importantly, recent findings show higher level of plasma Chol in Alzheimer's disease patients compared to healthy controls [16]. Furthermore, Chol has been identified to be present in plaques in a 1:1 ratio with $A \beta[17,18]$. Other studies revealed that feeding a Chol-enriched diet to rats resulted in the enhancement of APP, A $\beta$, and p-tau in the cortex region, which was associated with cognitive problems [19]. In a different study, it was observed that a Chol-rich diet increased the brain Chol level and resulted in motor function impairment [20]. Furthermore, neuronal Chol content has been linked with age, with higher Chol concentration being found in mature neurons compared to younger [21]. Together these results clearly connect Chol with disease development; however, the molecular mechanism of how Chol affects disease development remains unknown.

Aggregates extracted from patient brains have revealed the existence of oligomerlipid ensembles, pointing to possible direct interaction of free lipids with $\mathrm{A} \beta[22,23]$. Additionally, recent studies [24] have reported assemblies of $A \beta(1-42)$ monomers with Chol. These reports lead us to posit that free lipids affect the aggregation of amyloid proteins. Here we tested the hypothesis on the role of free $\mathrm{Chol}$ in the aggregation of $\mathrm{A} \beta$, at the physiologically relevant nanomolar concentration. Time-lapse Atomic Force Microscopy (AFM) was applied to monitor the in-situ formation of $A \beta(1-42)$ aggregates on supported lipid bilayers in the presence of free Chol. These studies revealed that $A \beta(1-42)$ aggregates are formed more rapidly on the lipid bilayer in presence of free Chol. Furthermore, the aggregation kinetics of $A \beta$ in the presence of free $C h o l$ is greatest on bilayers containing $\mathrm{Chol}$. Moreover, in the presence of free $\mathrm{Chol}$, aggregates accumulate more rapidly in the bulk above the membrane bilayer. Altogether, these studies revealed a critical role of free Chol on the disease-prone aggregation of $A \beta(1-42)$, suggesting that Chol can be a trigger of the aggregation process.

\section{Results}

\subsection{Rapid Appearance of Aggregates in Presence of Free Cholesterol}

The role of free $\mathrm{Chol}$ in the aggregation of $\mathrm{A} \beta(1-42)$ was investigated on a supported lipid bilayer surface. Briefly, a mixed lipid bilayer (PC-PS), containing 1-palmitoyl-2-oleoylglycero-3-phosphocholine (PC) and 1-palmitoyl-2-oleoyl-sn-glycero-3-phospho-L-serine (PS), was prepared as described earlier [12]. Then, $10 \mathrm{nM} \mathrm{A} \beta(1-42)$ monomer solution with and without $100 \mathrm{nM}$ Chol was deposited on the bilayer and time-lapse AFM imaging was performed to visualize the on-surface aggregation process. 
Figure 1a shows the lipid bilayer surface before the addition of $A \beta$ solution. The surface is smooth and homogeneous, with no aggregate-like features or trapped vesicles, which is critical for monitoring the on-membrane aggregation events [25-27]. Aggregates were detected $1 \mathrm{~h}$ after the addition of the $A \beta$ solution and continued growing in numbers in the subsequent time-points of $3 \mathrm{~h}$ and $5 \mathrm{~h}$ (Figure $1 \mathrm{~b}, \mathrm{c}$ ). To quantify the aggregation process, the volume of the aggregates, at each timepoint, was measured (Figure $1 \mathrm{~d}$ ). The plot shows that the mean aggregate volume increases as a function of incubation time on the PC-PS lipid bilayer.
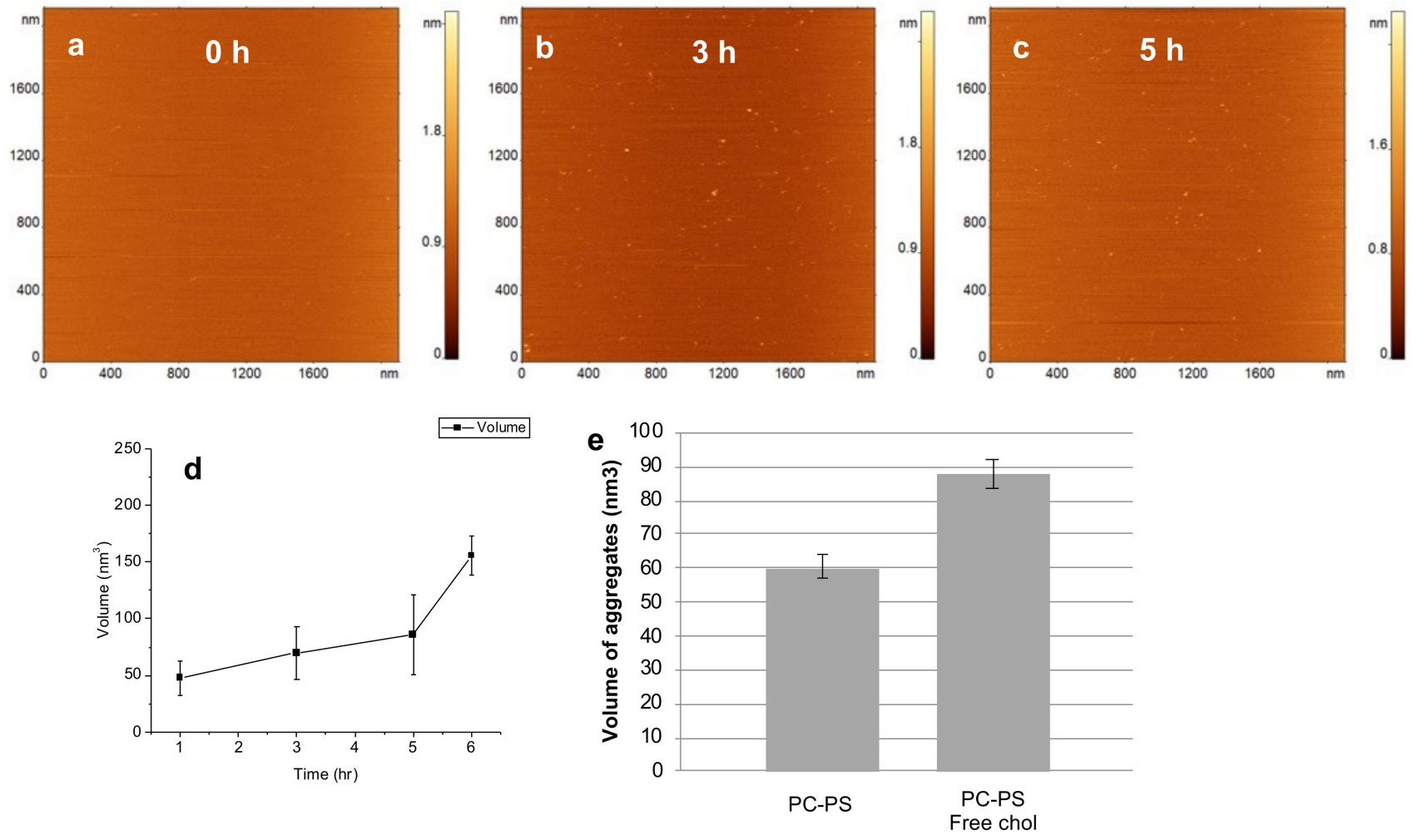

Figure 1. Aggregation of $10 \mathrm{nM} \mathrm{A} \beta(1-42)$, in the presence of $100 \mathrm{nM}$ Chol, on PC-PS lipid bilayer. (a) AFM image of the bilayer surface before addition of $A \beta(1-42)$-Chol solution. (b,c) AFM images of the same area of the lipid bilayer $3 \mathrm{~h}$ and $5 \mathrm{~h}$ after addition of $\mathrm{A} \beta(1-42)$-Chol solution. (d) Evolution of $A \beta(1-42)$ aggregate volume with time. (e) Comparison of $A \beta(1-42)$ aggregate volumes after $5 \mathrm{~h}$ incubation in the presence of PC-PS bilayer and PC-PS bilayer with Chol in solution. The volume of aggregates is significantly larger $(p<0.0001, t$-test $)$ in presence of free Chol.

As a control, we performed aggregation experiments by incubating $10 \mathrm{nM} A \beta(1-42)$ on the PC-PS bilayer without $\mathrm{Chol}$ in solution. Comparison of the volume of aggregates formed after $5 \mathrm{~h}$ incubation, with and without Chol present in the solution, is shown in Figure 1e. It is evident that aggregates are significantly larger when free $\mathrm{Chol}$ is present in the solution during aggregation, compared to only the $A \beta(1-42)$ in solution.

\subsection{Acceleration of A (1-42) Aggregation by Cholesterol inside Membrane}

To understand if the bilayer composition is important during aggregation with free Chol in solution, we assembled a mixed bilayer with Chol, PC-PS-Chol bilayer, and followed the aggregation of $A \beta$ in the presence of free $C$ hol on this bilayer. Representative time-lapse AFM imaging data are shown in Figure 2 and Figure S1. Initially, the bilayer surface is smooth, Figure S1a. Aggregates appear within 30 min of A $\beta$-Chol solution addition; a few are highlighted with white arrows in Figure S1b. After $2 \mathrm{~h}$ of incubation, the lipid bilayer surface shows a significant number of large aggregates (Figure 2a). Quantitative volume 
measurements for the two time-points show the change in aggregate size (Figure S1c,d). The aggregate size increased approximately 4 times, from $\sim 65 \mathrm{~nm}^{3}$ to $\sim 272 \mathrm{~nm}^{3}$, between $30 \mathrm{~min}$ and $2 \mathrm{~h}$.
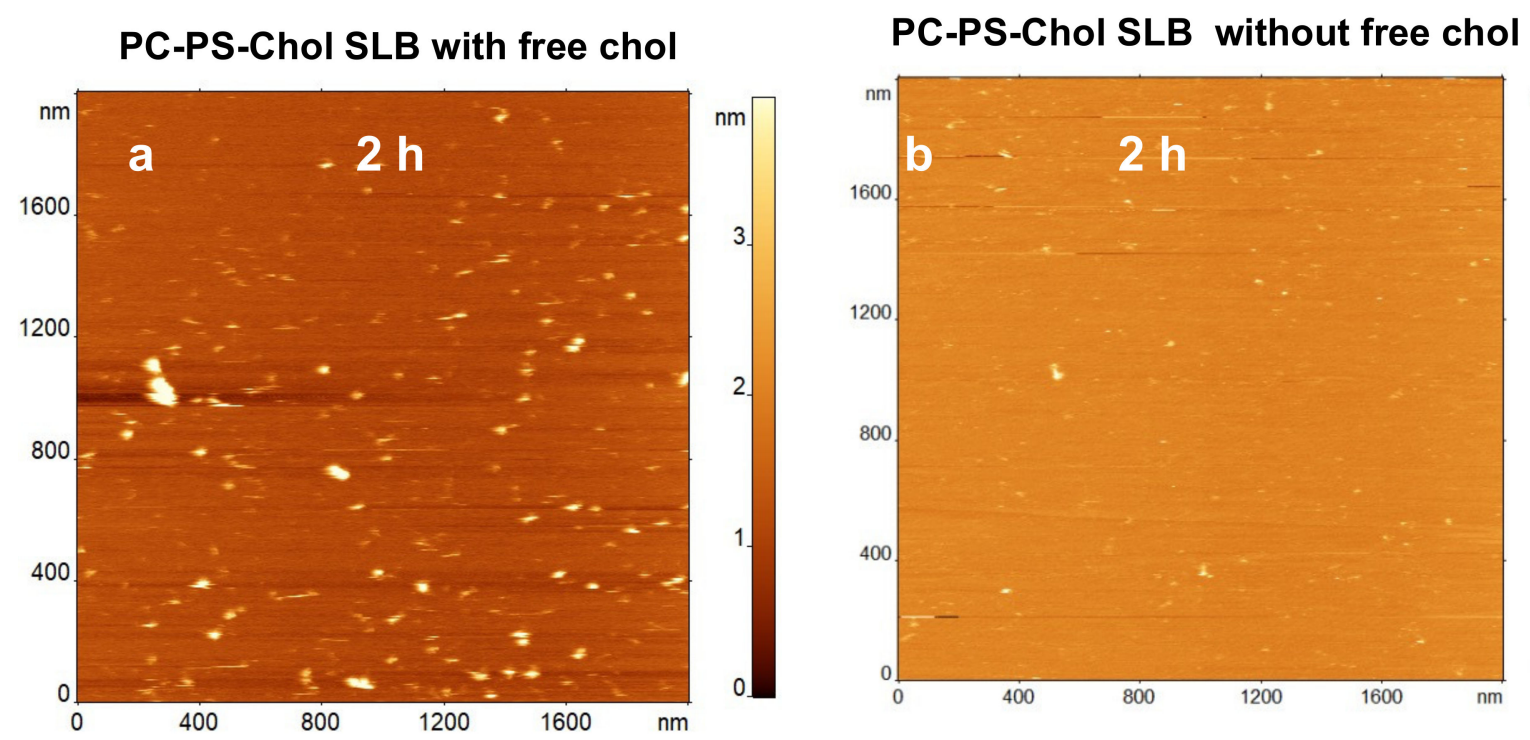

\section{Volume of aggregates $\left(\mathrm{nm}^{3}\right)$}
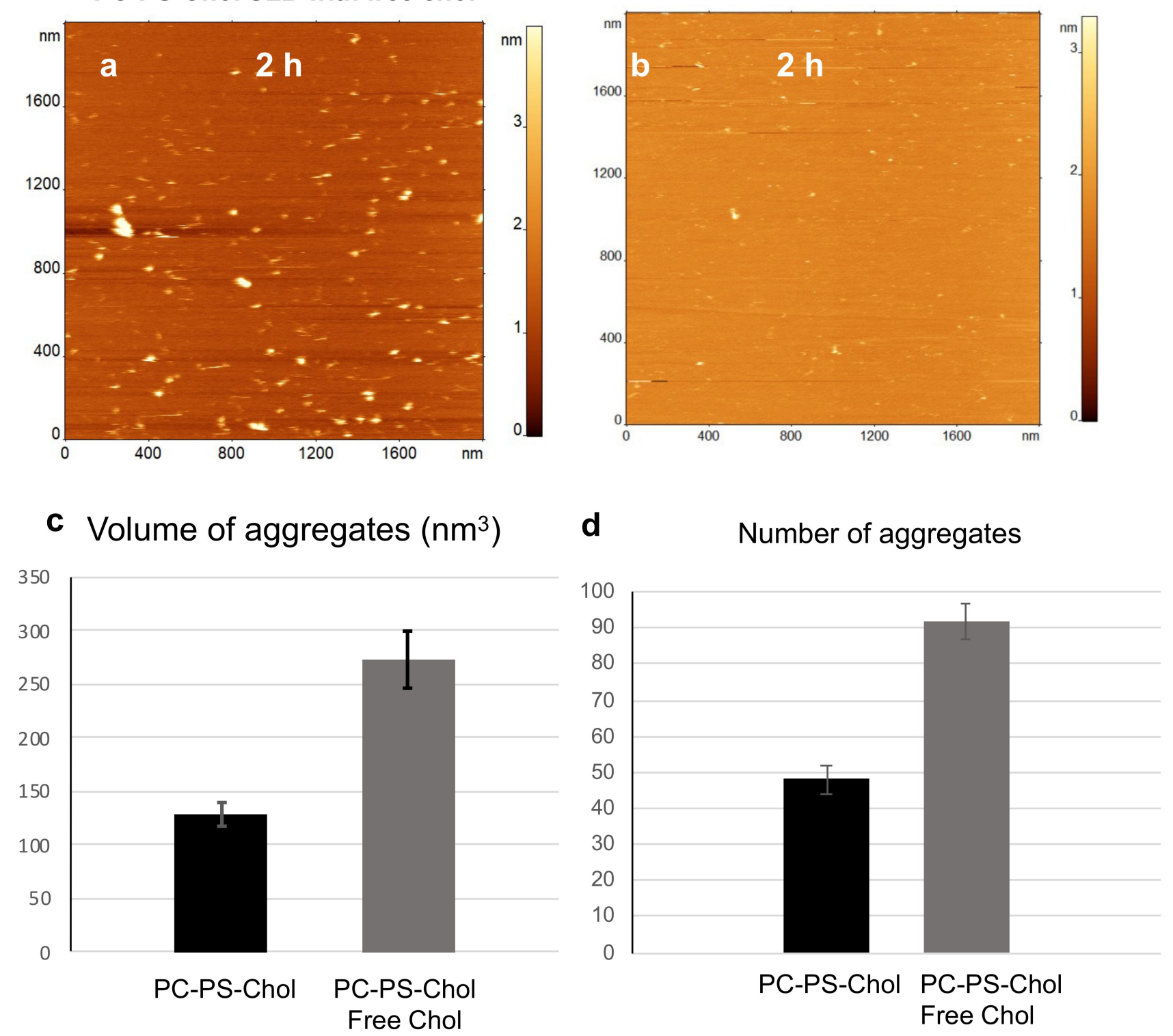

Figure 2. Aggregation of $10 \mathrm{nM} A \beta(1-42)$ on PC-PS-Chol bilayer. (a) AFM image of the PC-PS-Chol lipid bilayer after $2 \mathrm{~h}$ incubation with $10 \mathrm{nM} \mathrm{A} \beta 42$ and $100 \mathrm{nM}$ Chol in the solution. (b) AFM image of similar aggregation experiment as (a), except the absence of $100 \mathrm{nM}$ Chol in the solution. (c) Comparison of the on-bilayer aggregate volumes in the two aggregation experiments. Data is the mean value of aggregate volumes, obtained through Gaussian fits. Presence of free Chol significantly increases ( $p=0.001, t$-test) oligomer volume. (d) Comparison of the number of aggregates formed on the lipid bilayers in the presence and absence of $\mathrm{Chol}$ in solution; presence of free Chol leads to significantly more oligomers ( $p=0.003, t$-test). For $(\mathbf{c})$ and $(\mathbf{d})$ the error bars represent the standard error of the mean. 
We then performed aggregation experiments with only $A \beta(1-42)$ in solution in the presence of a PC-PS-Chol bilayer, Figure $2 b$. Visually it is evident that greater number of aggregates are present when free $\mathrm{Chol}$ is in the solution. Quantitative analysis of the two experiments shows that the volume as well as the total number of aggregates are significantly greater when $A \beta(1-42)$ aggregates in presence of free Chol in solution, Figure 2c,d.

To validate the observations and to test whether Chol itself can form aggregate-like features on the bilayer surface, we performed time-lapse experiments on the PC-PS-Chol bilayer in presence of Chol only. Figure S2a shows a large area of the bilayer surface prior to addition of Chol solution. Figure S2b, shows a zoom of the same area after $2 \mathrm{~h}$ incubation with Chol solution. Figure S2b,c shows another area on the bilayer surface after $2 \mathrm{~h}$ incubation with Chol solution; there are no aggregates or aggregate-like features on the surface of the bilayer. These observations clearly demonstrate that the aggregates, which were observed on the bilayer surface, were indeed self-assembled $A \beta(1-42)$ oligomers and that Chol inside the membrane works in synergy with free Chol, catalyzing the selfassembly of amyloid oligomers.

\subsection{Dynamics of $A \beta(1-42)$ Aggregation in Presence of Free Cholesterol}

After $2 \mathrm{~h}$ aggregation of $\mathrm{A} \beta$-Chol solution on the PC-PS-Chol bilayer, the surface is practically covered with aggregates, Figure $2 \mathrm{a}$. However, at $3 \mathrm{~h}$ significantly fewer aggregates are observed, Figure S3a. While the number of aggregates become fewer with increased aggregation time, their volumes increase, Figure S3b-d. Volume measurements of the aggregates after $1 \mathrm{~h}$ incubation show, Figure S3b, that the aggregate volumes are centered around $74 \mathrm{~nm}^{3}$. As the aggregates become larger at $3 \mathrm{~h}$, the distribution changes, and a peak around $293 \mathrm{~nm}^{3}$ becomes prominent. Larger aggregates also appear, Figure S3c. At the $4 \mathrm{~h}$ incubation point the aggregates are significantly larger, with a peak around $397 \mathrm{~nm}^{3}$, Figure S3d.

Previous studies [12] have shown that aggregates are capable of dissociating from the bilayer surface. Aggregates in the presence of free Chol show similar behavior, and the findings suggest that the presence of $\mathrm{Chol}$ in the solution accelerates the dissociation of aggregates. This phenomenon was tested by characterizing the accumulation of aggregates in the bulk solution above the bilayer using AFM. In these experiments, $10 \mathrm{nM} \mathrm{A} \beta(1-42)$ with $100 \mathrm{nM}$ Chol solution was incubated on top of PC-PS-Chol bilayer surface. At certain time intervals an aliquot was taken from the bulk solution above the bilayer, deposited onto APS-functionalized mica, and characterized using AFM imaging. The data is assembled in Figure 3. Aggregates, accumulated in the bulk solution above the bilayer, were detected after $3 \mathrm{~h}$, Figure $3 \mathrm{a}$, and become more prominent after $6 \mathrm{~h}$, Figure $3 \mathrm{~b}$. At the same time, control experiments conducted with $\mathrm{A} \beta(1-42)$ and $\mathrm{Chol}$ without the bilayer present show a negligible number of aggregates, Figure $3 \mathrm{c}$. Volumes of the aggregates were also analyzed and show that the average size of the aggregates increases over time, Figure $3 \mathrm{~d}$. These results show that the aggregates, which dissociate from the surface, do accumulate in the bulk solution, increasing the level of soluble aggregates. The data also show that, compared with the control experiments, in which $10 \mathrm{nM} \mathrm{A} \beta(1-42)$ and $100 \mathrm{nM}$ Chol were incubated without the bilayer, the presence of the bilayer leads to statistically significant more accumulation of aggregates in the bulk solution. 
a

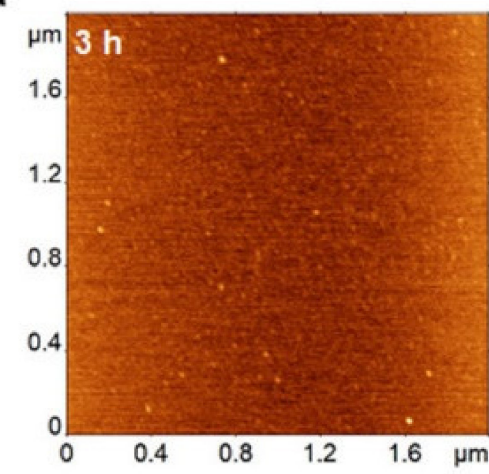

C

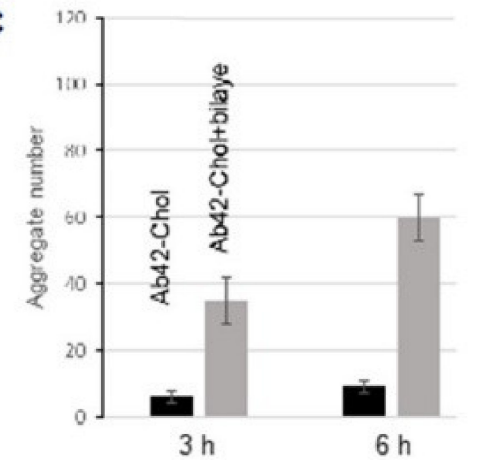

b
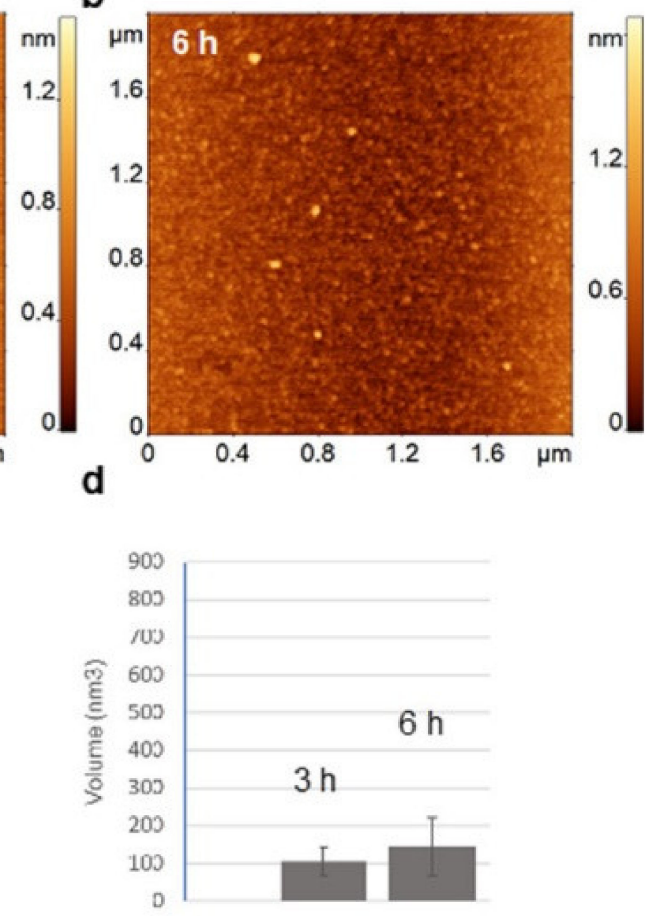

Figure 3. A $\beta(1-42)$ aggregate desorption from PC-PS-Chol lipid bilayer in presence of free Chol. $(\mathbf{a}, \mathbf{b})$ AFM images of aggregates from aliquots taken from the solution above the PC-PS-Chol bilayer while $10 \mathrm{nM} \mathrm{A} \beta(1-42)$ and $100 \mathrm{nM}$ Chol was incubating. Samples were taken $3 \mathrm{~h}$ and $6 \mathrm{~h}$ after addition of $A \beta(1-42)$-Chol solution. (c) Comparison of aggregates after $3 \mathrm{~h}$ and $6 \mathrm{~h}$ incubation of $\mathrm{A} \beta(1-42)$-Chol in the absence and presence of PC-PS-Chol bilayer. Presence of free Chol significantly increases number of desorbed oligomers, furthermore the increase from $3 \mathrm{~h}$ to $6 \mathrm{~h}$ time point is also significant ( $p=0.009, t$-test). (d) Comparison of aggregate volumes formed in presence of free Chol, depicted in (c).

\subsection{Computer Simulation of Interactions of $A \beta(1-42)$ with Free Cholesterol}

We used all-atom molecular dynamics simulations to elucidate the interaction of free Chol with $A \beta(1-42)$ monomers. Briefly, monomeric $A \beta(1-42)$ was placed in an explicit water box, and $\mathrm{NaCl}$ ions were used to neutralize the system charge and keep the ionic strength at a physiologically relevant concentration, $150 \mathrm{mM}$. A $\beta(1-42)$ was placed at $4 \mathrm{~nm}$ from a single Chol molecule. Dynamics of $A \beta(1-42)$ without Chol was simulated as a control. Five replicas of each simulation system were run for $10 \mu \mathrm{s}$, yielding a cumulative simulation time of $50 \mu$ s for each system.

The $A \beta(1-42)$ monomer shows a rough free energy landscape (FEL), calculated using dihedral principle component analysis of the concatenated dataset, when in the presence of a single free Chol molecule, Figure 4a. The FEL contains well-separated energy minima in three distinct areas, two small areas to the upper and lower left, and a single, large, rough area to the right. The 10 lowest energy minima are highlighted in Figure 4a, and the representative structure for each cluster of said minima are also presented, showing the Chol molecule. These 10 clusters represent $\sim 45.6 \%$ of the conformations sampled during the simulation. The number of protein residues in contact with Chol plotted versus the simulation time are given for each individual simulation run in Figure S4a-e. It is evident that the Chol molecule does not simultaneously interact with many residues of $A \beta(1-42)$ at any given time. In fact, the majority of interactions occur through contacts with single residues. Quantitative analysis of these data show that specific regions of $\mathrm{A} \beta(1-42)$ are more likely to interact with the $\mathrm{Chol}$ molecule, Figure $4 \mathrm{~b}$. The contact probability for each residue, based on the combined $50 \mu$ s dataset, shows that residues 10 through 14 are most 
likely to interact with Chol, followed by residues 1-8 of the N-terminal region. Residues in the central hydrophobic region (CHC, residues 17-21) are also likely interaction partners, albeit with lower probability than the aforementioned regions.

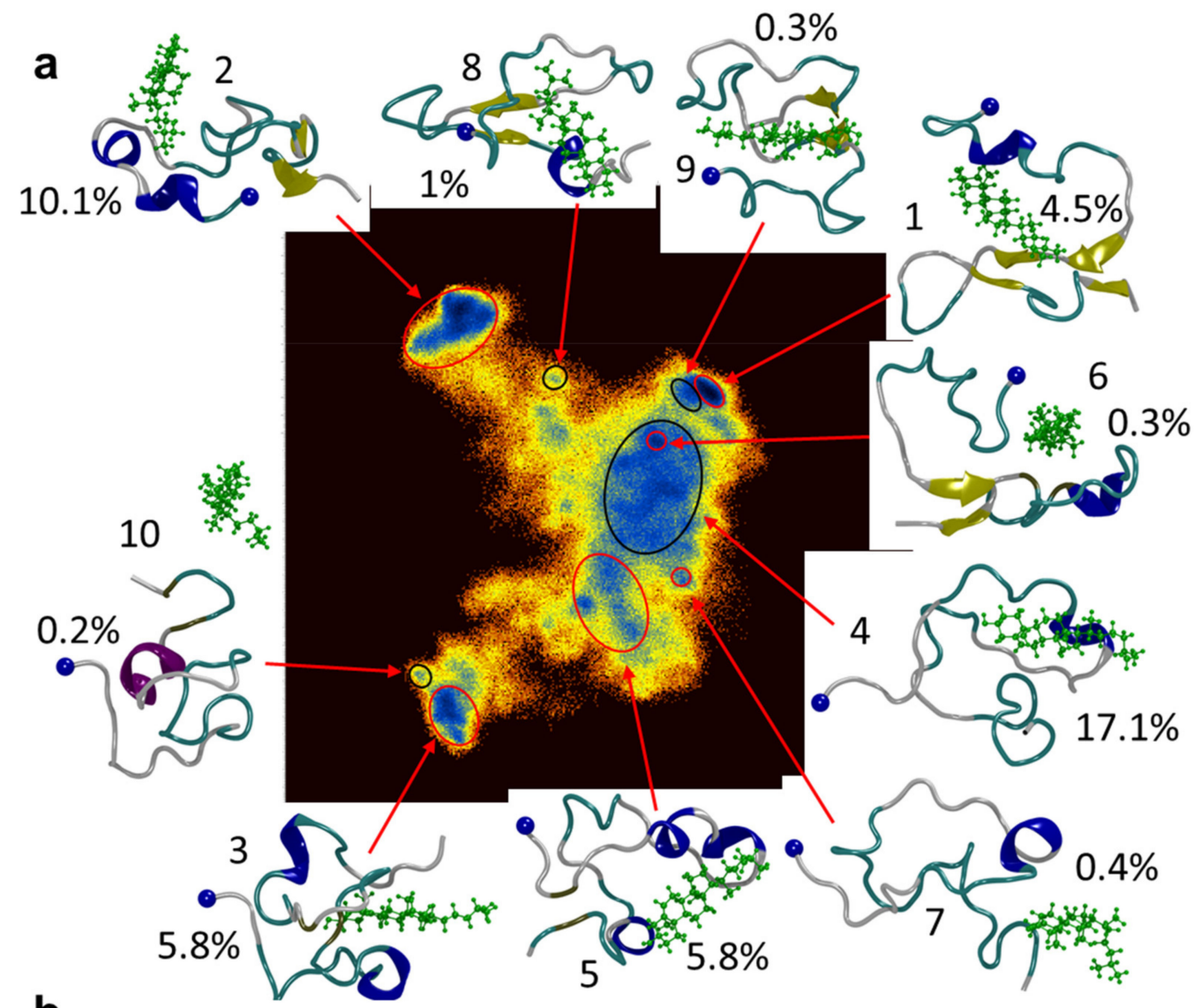

b

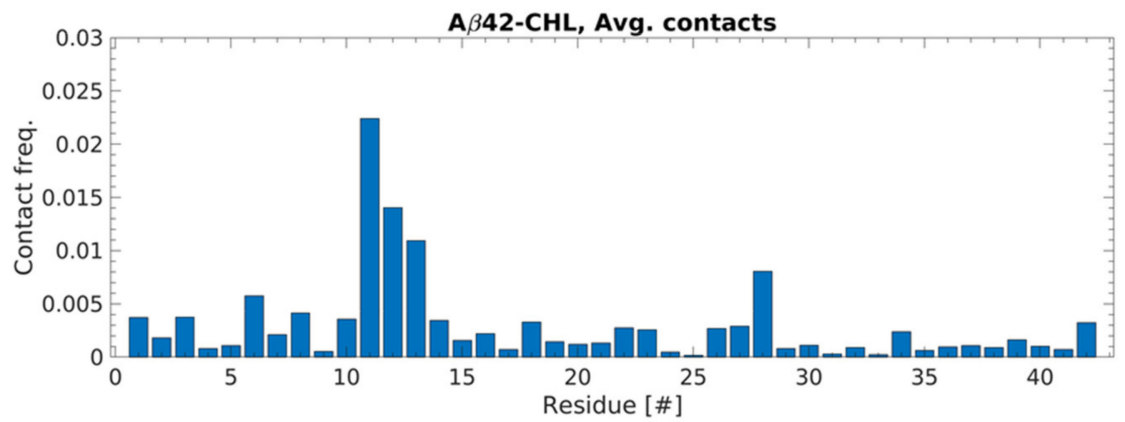

Figure 4. MD simulation of $\mathrm{A} \beta(1-42)$ interacting with Chol. (a) Free energy landscape based on dihedral principal component analysis of cumulative $50 \mu$ s simulation of $A \beta(1-42)$ interacting with Chol. The 10 lowest energy minima are highlighted and the representative conformation of the $\mathrm{A} \beta(1-42)$ is shown. Percentages indicate the fraction of conformations relative to total number sampled during the simulations. Blue sphere denotes the N-terminal. (b) Average contact probability between residues of $\mathrm{A} \beta 42$ and the Chol molecule.

$\mathrm{A} \beta(1-42)$ monomer, in the absence of Chol, shows a dramatically different FEL, Figure 5, in which the deepest energy minimum is isolated and dominates by number of conformations $(\sim 11.7 \%)$ while the rest of the minima are scattered around a very rough area. Furthermore, the 10 lowest energy clusters only represent $\sim 19.9 \%$ of the conformations sampled during the simulations. Comparing the evolution of secondary structure for the different simulations, Figures S5 and S6, shows that in both systems the A $\beta(1-42)$ monomer 
is dominated by turn/bend conformations, with gradual increases in $\beta$-strand structure for each system. However, interactions with $\mathrm{Chol}$ seems to hinder the formation of long-lived $\beta$-strands, as in 3/5 of simulations $\beta$-strand appear and disappear more rapidly than in the control simulations without Chol, Figure S5 compared to Figure S6.

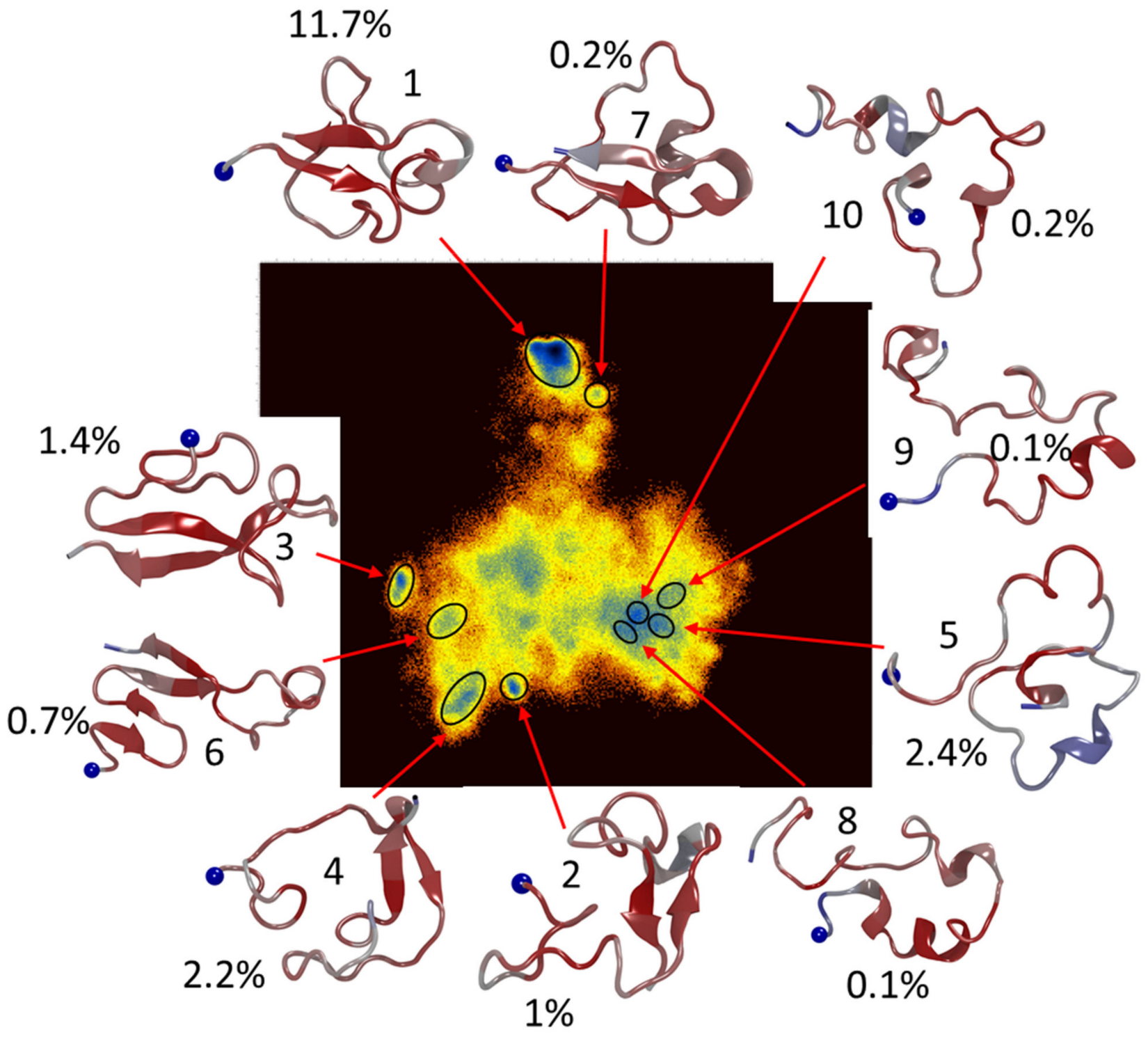

Figure 5. MD simulation of $A \beta(1-42)$ monomer. Free energy landscape based on dihedral principal component analysis of cumulative $50 \mu$ s simulation of $A \beta(1-42)$ monomer. The 10 lowest energy minima are highlighted and the representative conformation of the $A \beta(1-42)$ is shown; colors indicate degree of fluctuation in structure, with red being highly conserved regions and blue being highly dynamic regions. Percentages indicate the fraction of conformations relative to total number sampled during the simulations. Blue sphere denotes the N-terminal.

\section{Discussion}

In our previous study, we have shown that the presence of Chol in the lipid bilayer facilitates aggregation of $A \beta(1-42)$ leading to rapid formation of aggregates [12]. The number of aggregates formed in presence of Chol-containing bilayers was 6 times greater compared to the aggregates on bilayers devoid of Chol. These results revealed the critical role of $\mathrm{Chol}$ in the aggregation process. Here, we have shown that free $\mathrm{Chol}$, in addition to Chol inside the lipid bilayer (PC-PS-Chol), has an accelerating effect on A $\beta(1-42)$ aggregation. 
Results unambiguously show that free Chol can further accelerate $A \beta(1-42)$ aggregation, as the size and number of aggregates formed in presence of free Chol are greater compared to the experiments where it is absent (Figures 1 and 2). This enhanced effect of free Chol indicates the possibility of direct interaction between $C$ hol and $A \beta(1-42)$. Several studies have shown this type of direct binding, among them [28]. NMR studies have revealed Chol-binding regions of $C 99$, which is the source of $A \beta$ peptide generation due to the action of $\gamma$-secretase. The region encompassing residues $18-40$ of $A \beta(1-42)$ is observed to interact with Chol [29]. Furthermore, insertion studies of various length of peptide fragments such as $A \beta(17-40), A \beta(22-35), A \beta(25-35)$ have shown that fragments containing residues 25-35 successfully penetrated the Chol containing monolayer [30].

The findings on direct binding of free Chol to $A \beta$ monomers are in line with our all-atom simulations (Figures 4 and 5). Moreover, the energy landscapes qualitatively support the observation of increased dynamics in the $A \beta$ molecule in the presence of Chol (Figure 4). The presence of Chol dramatically increases the sampling of the free energy landscape, but more importantly also increases the number of sampled low-energy conformations. The 10 lowest energy minima sampled by the $A \beta(1-42)$ monomer, in presence of Chol, make up almost $46 \%$ of total conformations sampled during the $50 \mu \mathrm{s}$ cumulative simulations. At the same time, in the absence of Chol, the 10 lowest minima make up almost $20 \%$ of the sampled conformations. This acceleration of conformational search may be the key for how Chol affects the aggregation. Indeed, comparing interactions with membranes with and without Chol showed that the $A \beta(1-42)$ monomer experiences a similar increased sampling when Chol is present in the membrane [12]. Additionally, the affinity of the monomer to the membrane is also changed by Chol [12,31]. Furthermore, the simulations show that dimer formation on membranes with Chol inside occur almost $2 X$ faster than on a similar membrane without $\mathrm{Chol}$ [12]. The effect of Chol on the free energy and conformational sampling has also been reported for $\mathrm{A} \beta$ dimers and trimers [32]. In addition to significant changes to the FEL, the authors also report that presence of Chol induces greater $\beta$-structure content in the dimers and trimers of the $A \beta(1-42)$; they also report that dimer to trimer change in $\beta$-structure is also significant when $\mathrm{Chol}$ is present, going from $26 \%$ to $41 \%$ [32]. The discrepancy in fraction of $\beta$-structure secondary structure between monomer and oligomers can be explained by data obtained by Ono et al., in which different pure oligomers of defined sizes were compared [33]. They reported that oligomer size has a significant effect on the structure and that there is a significant alteration of the A $\beta$ structure going from monomer to dimer.

Our results, demonstrating the accelerating effect of free Chol on $A \beta(1-42)$ aggregation, directly suggest that interference or blocking of Chol-A $\beta$ interaction may suppress spontaneous self-assembly of the protein and thereby reduce the early-stage toxic oligomers. Studies following this line of thought have shown promising results. Bexarotene, which binds to the Chol-binding domain of $\mathrm{A} \beta$, poses a competition for Chol towards $\mathrm{A} \beta[34,35]$. Treatment with nanomolar concentration of bexarotene prevented $A \beta$ oligomer induced $\mathrm{Ca}^{2+}$ flux. These data indicate that the prevention of direct interaction of Chol with $\mathrm{A} \beta$ can significantly reduce the toxicity caused by the oligomers [34].

One of the important findings in the present study is the increased aggregate dynamics caused by the presence of free Chol (Figure 3 and Figure S3). The data shows that, although aggregates are rapidly formed on the surface, they are not firmly attached to the bilayer and can easily leave the surface spontaneously. This hypothesis is supported by a gradual accumulation of aggregates in the bulk solution above the membrane surface (Figure 3). These data clearly show that the bilayer surface, along with the presence of free Chol, can act as a highly efficient platform for producing oligomers, which then can either participate in further aggregation or act as toxic agents. Most notable, this efficient oligomer producing process occurs at physiologically low nanomolar concentrations of $A \beta(1-42)$.

Another aspect of the oligomers formed in the presence of free Chol is their greater size compared to those formed in the absence of free Chol. Yasumoto et al. reported that low- (LMW) and high-molecular weight (HMW) oligomers use different pathways to 
damage neurons, with HMW being more neurotoxic and causing more direct damage to the membranes [36]. In particular, HMW oligomers caused significantly more membrane depolarization and impaired long-term potentiation. In the context of the current study, large oligomers, produced due to interactions with free $\mathrm{Chol}$, that dissociated from the membrane surface may show similar mechanism of action as the HMW oligomers tested in the aforementioned study.

Overall, the present study shows that the presence of free Chol, along with inmembrane Chol, significantly accelerates the $A \beta(1-42)$ aggregation. This process occurs at physiologically relevant conditions, including the low nanomolar protein concentration. These findings suggests that specific lipid-A $\beta$ interactions are critical factors for the spontaneous formation of neurotoxic oligomers. These findings further extend our model on the critical role of membrane composition in the assembly of disease-prone amyloid aggregates [12]. Our new data suggest that free $\mathrm{Chol}$ facilitate the aggregation process of $A \beta$ monomers. Importantly, there is a strong synergy between the in-membrane and free Chol in this membrane mediated catalysis of $A \beta$ aggregation at physiologically relevant conditions. Note a recent publication [37], which found accumulation of free Chol in the brain for a neurovisceral Niemann-Pick type C (NPC) disease. These findings suggest that the effects of free Chol and other lipids may also be extended to other diseases. Further neurotoxic studies of nanoaggregates assembled on the membranes, in parallel with structural characterization of such aggregates, will pave the way for the development of novel diagnostic and therapeutic strategies for $\mathrm{AD}$ and can be extended to other neurodegenerative diseases associated with the formation of protein deposits.

\section{Materials and Methods}

\subsection{Materials}

Lipids were purchased from Avanti Polar Lipids, Inc. (Alabama, US). A $\beta(1-42)$ was bought from AnaSpec (Fremont, CA, USA). Chloroform was procured from Sigma Aldrich Inc (St. Louis, MO, USA). The buffer solution that was used in this study is $20 \mathrm{mM}$ HEPES, $150 \mathrm{mM} \mathrm{NaCl}, 10 \mathrm{mM} \mathrm{CaCl}_{2}$, pH 7.4. All other chemicals, unless otherwise specified, were procured from Sigma at analytical chemistry grade or better.

\subsection{Preparation of Supported Lipid Bilayer}

PC-PS-Chol lipid bilayer was prepared on mica substrate as mentioned in the previous publication [12]. Briefly, POPC, POPS, and Chol vesicles were prepared by sonicating the mixture for $45 \mathrm{~min}$ until the mixture became clear and then deposited onto freshly cleaved mica surface attached to a glass slide. The slide was then incubated at $60^{\circ} \mathrm{C}$ for $1 \mathrm{~h}$. After the incubation, the sample was allowed to reach room temperature and then gently rinsed with a buffer containing $20 \mathrm{mM}$ HEPES, $150 \mathrm{mM} \mathrm{NaCl}$, pH 7.4. The bilayer was then imaged immediately by AFM in liquid.

\subsection{Preparation of $A \beta 42$ Protein Solution}

The method for preparing the $A \beta 42$ stock solution was kept similar to our previous publication [12]. Briefly, lyophilized A $\beta(1-42)$ was dissolved in $100 \mu \mathrm{L}$ of 1,1,1,3,3,3hexafluoroisopropanol (HFIP) at room temperature with sonication. The HFIP was then evacuated completely in a vacufuge. Anhydrous DMSO was then added to prepare the stock solution, which was then kept at $-20^{\circ} \mathrm{C}$. The stock solution was diluted in the buffer solution to prepare working solutions at the necessary concentrations. Working solutions were used immediately and leftover was discarded.

\subsection{Time-Lapse AFM Imaging}

Time-lapse data were obtained using an MFP-3D instrument (Asylum Research, Santa Barbara, CA, USA). AFM imaging, in buffer medium, was carried out in tapping mode using the cantilever "E" of MSNL probes (Bruker, Santa Barbara, CA, USA). The typical 
resonance frequency of the cantilever in buffer was 7-9 $\mathrm{kHz}$ with typical spring constants of $\sim 0.1 \mathrm{~N} / \mathrm{m}$. Scan speed was typically between 1 to $2 \mathrm{~Hz}$.

At the start of each time-lapse experiment the lipid bilayer was imaged to ensure a homogenous and smooth surface, devoid of any unruptured vesicles. A $\beta$ solution was then added, and time-lapse imaging commenced in the same area of the bilayer. The cantilever was parked after recording each frame to ensure that no damage to the lipid bilayer surface occurred due to scanning.

\subsection{AFM Data Analysis}

The presented AFM images have undergone minimal processing. Flattening was applied to the images (fitted with 1st order polynomial) with FemtoScan software (Advanced Technologies Center, Moscow, Russia). Grain analysis tool in the software was applied to measure the volume of the oligomers. The volume data were plotted as histograms using Origin Pro software (OriginLab, Northampton, MA, USA) and fitted with Gaussian distribution. The mean value of the oligomer volume for each time point was determined using the peak value of the distribution and the error bars represent the standard deviation, unless otherwise mentioned.

\subsection{Molecular Dynamics Simulations}

To investigate the interaction of $A \beta(1-42)$ monomer with Chol, we placed an $A \beta(1-42)$ monomer (conformation taken from [38]) at $4 \mathrm{~nm}$ center-of-mass (CoM) from a single Chol molecule, solvated the system in TIP3P water, neutralized with $\mathrm{NaCl}$ counter ions, and maintained a final $\mathrm{NaCl}$ concentration of $150 \mathrm{mM}$. Protein was described using the Amber ff99SB-ILDN force field [39], while Chol was described using the lipid17 force field (an extension and refinement of lipid14 [40]). A control system with only A $\beta(1-42)$ monomer was also created in a similar manner. The systems were then energy minimized, heated to $300 \mathrm{~K}$, and run for 500 ps as NVT ensemble. Production simulations were run as an NPT ensemble for $10 \mu \mathrm{s}$; simulations for each system were repeated five times for a total of $50 \mu$ s for each system. Simulations were performed using a 2 fs integration time step. The simulations employed periodic boundary conditions with an isotropic pressure coupling at 1 bar, a constant temperature of $300 \mathrm{~K}$, non-bonded interactions truncated at 10 $\AA$, and electrostatic interactions treated using particle-mesh Ewald [41]. Simulations were performed using the Amber18 package [42].

\subsection{Analysis of $M D$ Trajectories}

AmberTools20 suite of programs [43], Carma [44], and VMD [45] were used to analyze the obtained simulation trajectories. Graphs and mathematical analyses were obtained using MATLAB (MathWorks, Natick, MA, USA).

Supplementary Materials: The following supporting information can be downloaded at: https: //www.mdpi.com/article/10.3390/ijms23052803/s1.

Author Contributions: Y.L.L., S.B. and M.H. designed the project. S.B. performed the AFM experiments. M.H. performed and analyzed the molecular dynamics simulations. All authors have read and agreed to the published version of the manuscript.

Funding: This research was funded by National Institutes of Health, grants GM096039 and GM118006 to Y.L.L.

Institutional Review Board Statement: Not applicable.

Informed Consent Statement: Not applicable.

Data Availability Statement: The data that support the findings of this study are available from the corresponding author upon reasonable request. 


\begin{abstract}
Acknowledgments: Anton 2 computer time was provided by the Pittsburgh Supercomputing Center (PSC) through Grant R01GM116961 from the National Institutes of Health. The Anton 2 machine at PSC was generously made available by D.E. Shaw Research. This work was completed utilizing the Holland Computing Center of the University of Nebraska, which receives support from the Nebraska Research Initiative. Authors thank Thomas D. Stormberg for proof reading.
\end{abstract}

Conflicts of Interest: The authors declare no conflict of interest.

\title{
References
}

1. Ross, C.A.; Poirier, M.A. Protein aggregation and neurodegenerative disease. Nat. Med. 2004, 10, S10-S17. [CrossRef] [PubMed]

2. Chiti, F.; Dobson, C.M. Protein Misfolding, Amyloid Formation, and Human Disease: A Summary of Progress Over the Last Decade. Annu. Rev. Biochem. 2017, 86, 27-68. [CrossRef] [PubMed]

3. Hardy, J.A.; Higgins, G.A. Alzheimer's disease: The amyloid cascade hypothesis. Science 1992, 256, 184. [CrossRef] [PubMed]

4. Hardy, J. Has the Amyloid Cascade Hypothesis for Alzheimers Disease been Proved? Curr. Alzheimer Res. 2006, 3, 71-73. [CrossRef] [PubMed]

5. Hu, X.; Crick, S.L.; Bu, G.; Frieden, C.; Pappu, R.V.; Lee, J.M. Amyloid seeds formed by cellular uptake, concentration, and aggregation of the amyloid-beta peptide. Proc. Natl. Acad. Sci. USA 2009, 106, 20324-20329. [CrossRef]

6. Grimmer, T.; Riemenschneider, M.; Förstl, H.; Henriksen, G.; Klunk, W.E.; Mathis, C.A.; Shiga, T.; Wester, H.-J.; Kurz, A.; Drzezga, A. Beta Amyloid in Alzheimer's Disease: Increased Deposition in Brain Is Reflected in Reduced Concentration in Cerebrospinal Fluid. Biol. Psychiatry 2009, 65, 927-934. [CrossRef]

7. Banerjee, S.; Hashemi, M.; Lv, Z.; Maity, S.; Rochet, J.C.; Lyubchenko, Y.L. A novel pathway for amyloids self-assembly in aggregates at nanomolar concentration mediated by the interaction with surfaces. Sci. Rep. 2017, 7, 45592. [CrossRef]

8. Pan, Y.; Banerjee, S.; Zagorski, K.; Shlyakhtenko, L.S.; Kolomeisky, A.B.; Lyubchenko, Y.L. Molecular Model for the SurfaceCatalyzed Protein Self-Assembly. J. Phys. Chem. B 2019, 124, 366-372. [CrossRef]

9. Matsubara, T.; Nishihara, M.; Yasumori, H.; Nakai, M.; Yanagisawa, K.; Sato, T. Size and Shape of Amyloid Fibrils Induced by Ganglioside Nanoclusters: Role of Sialyl Oligosaccharide in Fibril Formation. Langmuir 2017, 33, 13874-13881. [CrossRef]

10. Chi, E.Y.; Frey, S.L.; Lee, K.Y.C. Ganglioside GM1-Mediated Amyloid-beta Fibrillogenesis and Membrane Disruption. Biochemistry 2007, 46, 1913-1924. [CrossRef]

11. Matsubara, T.; Yasumori, H.; Ito, K.; Shimoaka, T.; Hasegawa, T.; Sato, T. Amyloid- $\beta$ fibrils assembled on ganglioside-enriched membranes contain both parallel $\beta$-sheets and turns. J. Biol. Chem. 2018, 293, 14146-14154. [CrossRef] [PubMed]

12. Banerjee, S.; Hashemi, M.; Zagorski, K.; Lyubchenko, Y.L. Cholesterol in Membranes Facilitates Aggregation of Amyloid $\beta$ Protein at Physiologically Relevant Concentrations. ACS Chem. Neurosci. 2021, 12, 506-516. [CrossRef] [PubMed]

13. Hicks, D.A.; Nalivaeva, N.N.; Turner, A.J. Lipid Rafts and Alzheimer's Disease: Protein-Lipid Interactions and Perturbation of Signaling. Front. Physiol. 2012, 3, 189. [CrossRef] [PubMed]

14. Ahyayauch, H.; de la Arada, I.; Masserini, M.E.; Arrondo, J.L.R.; Goñi, F.M.; Alonso, A. The Binding of A $\beta 42$ Peptide Monomers to Sphingomyelin/Cholesterol/Ganglioside Bilayers Assayed by Density Gradient Ultracentrifugation. Int. J. Mol. Sci. 2020, 21, 1674. [CrossRef]

15. Fantini, J.; Yahi, N. Molecular insights into amyloid regulation by membrane cholesterol and sphingolipids: Common mechanisms in neurodegenerative diseases. Expert Rev. Mol. Med. 2010, 12, e27. [CrossRef]

16. Popp, J.; Meichsner, S.; Kölsch, H.; Lewczuk, P.; Maier, W.; Kornhuber, J.; Jessen, F.; Lütjohann, D. Cerebral and extracerebral cholesterol metabolism and CSF markers of Alzheimer's disease. Biochem. Pharmacol. 2013, 86, 37-42. [CrossRef]

17. Panchal, M.; Loeper, J.; Cossec, J.-C.; Perruchini, C.; Lazar, A.; Pompon, D.; Duyckaerts, C. Enrichment of cholesterol in microdissected Alzheimer's disease senile plaques as assessed by mass spectrometry. J. Lipid Res. 2010, 51, 598-605. [CrossRef]

18. Gellermann, G.P.; Appel, T.R.; Davies, P.; Diekmann, S. Paired helical filaments contain small amounts of cholesterol, phosphatidylcholine and sphingolipids. Biol. Chem. 2006, 387, 1267-1274. [CrossRef]

19. Ullrich, C.; Pirchl, M.; Humpel, C. Hypercholesterolemia in rats impairs the cholinergic system and leads to memory deficits. Mol. Cell. Neurosci. 2010, 45, 408-417. [CrossRef]

20. Tansey, M.G.; Paul, R.; Choudhury, A.; Kumar, S.; Giri, A.; Sandhir, R.; Borah, A. Cholesterol contributes to dopamine-neuronal loss in MPTP mouse model of Parkinson's disease: Involvement of mitochondrial dysfunctions and oxidative stress. PLoS ONE 2017, 12, e0171285. [CrossRef]

21. Nicholson, A.M.; Ferreira, A. Increased Membrane Cholesterol Might Render Mature Hippocampal Neurons More Susceptible to ß-Amyloid-Induced Calpain Activation and Tau Toxicity. J. Neurosci. 2009, 29, 4640-4651. [CrossRef] [PubMed]

22. Kiskis, J.; Fink, H.; Nyberg, L.; Thyr, J.; Li, J.-Y.; Enejder, A. Plaque-associated lipids in Alzheimer's diseased brain tissue visualized by nonlinear microscopy. Sci. Rep. 2015, 5, 13489. [CrossRef] [PubMed]

23. Martins, I.C.; Kuperstein, I.; Wilkinson, H.; Maes, E.; Vanbrabant, M.; Jonckheere, W.; Van Gelder, P.; Hartmann, D.; D’Hooge, R.; De Strooper, B.; et al. Lipids revert inert A $\beta$ amyloid fibrils to neurotoxic protofibrils that affect learning in mice. EMBO 2007, 27, 224-233. [CrossRef] [PubMed]

24. Sparr, E.; Linse, S. Lipid-protein interactions in amyloid formation. Biochim. Biophys. Acta 2019, 1867, 455-457. [CrossRef] [PubMed] 
25. Banerjee, S.; Hashemi, M.; Zagorski, K.; Lyubchenko, Y.L. Interaction of A $\beta 42$ with Membranes Triggers the Self-Assembly into Oligomers. Int. J. Mol. Sci. 2020, 21, 1129. [CrossRef]

26. Lv, Z.; Banerjee, S.; Zagorski, K.; Lyubchenko, Y.L. Supported Lipid Bilayers for Atomic Force Microscopy Studies. Methods Mol. Biol. 2018, 1814, 129-143. [CrossRef]

27. Lv, Z.; Hashemi, M.; Banerjee, S.; Zagorski, K.; Rochet, J.-C.; Lyubchenko, Y.L. Assembly of $\alpha$-synuclein aggregates on phospholipid bilayers. Biochim. Biophys. Acta 2019, 1867, 802-812. [CrossRef]

28. Di Scala, C.; Chahinian, H.; Yahi, N.; Garmy, N.; Fantini, J. Interaction of Alzheimer's $\beta$-amyloid peptides with cholesterol: Mechanistic insights into amyloid pore formation. Biochemistry 2014, 53, 4489-4502. [CrossRef]

29. Barrett Paul, J.; Song, Y.; Van Horn Wade, D.; Hustedt Eric, J.; Schafer Johanna, M.; Hadziselimovic, A.; Beel Andrew, J.; Sanders Charles, R. The Amyloid Precursor Protein Has a Flexible Transmembrane Domain and Binds Cholesterol. Science 2012, 336, 1168-1171. [CrossRef]

30. Di Scala, C.; Yahi, N.; Lelièvre, C.; Garmy, N.; Chahinian, H.; Fantini, J. Biochemical Identification of a Linear Cholesterol-Binding Domain within Alzheimer's $\beta$ Amyloid Peptide. ACS Chem. Neurosci. 2013, 4, 509-517. [CrossRef]

31. Yu, X.; Zheng, J. Cholesterol Promotes the Interaction of Alzheimer $\beta$-Amyloid Monomer with Lipid Bilayer. J. Mol. Biol. 2012, 421, 561-571. [CrossRef] [PubMed]

32. Ngo, S.T.; Nguyen, P.H.; Derreumaux, P. Cholesterol Molecules Alter the Energy Landscape of Small A $\beta 1-42$ Oligomers. J. Phys. Chem. B 2021, 125, 2299-2307. [CrossRef] [PubMed]

33. Ono, K.; Condron, M.M.; Teplow, D.B. Structure-neurotoxicity relationships of amyloid $\beta$-protein oligomers. Proc. Natl. Acad. Sci. USA 2009, 106, 14745-14750. [CrossRef] [PubMed]

34. Fantini, J.; Di Scala, C.; Yahi, N.; Troadec, J.-D.; Sadelli, K.; Chahinian, H.; Garmy, N. Bexarotene Blocks Calcium-Permeable Ion Channels Formed by Neurotoxic Alzheimer's $\beta$-Amyloid Peptides. ACS Chem. Neurosci. 2014, 5, 216-224. [CrossRef] [PubMed]

35. Cramer Paige, E.; Cirrito John, R.; Wesson Daniel, W.; Lee, C.Y.D.; Karlo, J.C.; Zinn Adriana, E.; Casali Brad, T.; Restivo Jessica, L.; Goebel Whitney, D.; James Michael, J.; et al. ApoE-Directed Therapeutics Rapidly Clear $\beta$-Amyloid and Reverse Deficits in AD Mouse Models. Science 2012, 335, 1503-1506. [CrossRef] [PubMed]

36. Yasumoto, T.; Takamura, Y.; Tsuji, M.; Watanabe-Nakayama, T.; Imamura, K.; Inoue, H.; Nakamura, S.; Inoue, T.; Kimura, A.; Yano, S.; et al. High molecular weight amyloid $\beta$ 1-42 oligomers induce neurotoxicity via plasma membrane damage. FASEB J. 2019, 33, 9220-9234. [CrossRef] [PubMed]

37. Treiber-Held, S.; Distl, R.; Meske, V.; Albert, F.; Ohm, T.G. Spatial and temporal distribution of intracellular free cholesterol in brains of a Niemann-Pick type $\mathrm{C}$ mouse model showing hyperphosphorylated tau protein. Implications for Alzheimer's disease. J. Pathol. 2003, 200, 95-103. [CrossRef]

38. Zhang, Y.; Hashemi, M.; Lv, Z.; Lyubchenko, Y.L. Self-assembly of the full-length amyloid Abeta42 protein in dimers. Nanoscale 2016, 8, 18928-18937. [CrossRef]

39. Lindorff-Larsen, K.; Piana, S.; Palmo, K.; Maragakis, P.; Klepeis, J.L.; Dror, R.O.; Shaw, D.E. Improved side-chain torsion potentials for the Amber ff99SB protein force field. Proteins 2010, 78, 1950-1958. [CrossRef]

40. Dickson, C.J.; Madej, B.D.; Skjevik, Å.A.; Betz, R.M.; Teigen, K.; Gould, I.R.; Walker, R.C. Lipid14: The Amber Lipid Force Field. J Chem. Theory Comput. 2014, 10, 865-879. [CrossRef]

41. Darden, T.; York, D.; Pedersen, L. Particle mesh Ewald: An $N \cdot \log (\mathrm{N})$ method for Ewald sums in large systems. J. Chem. Phys. 1993, 98, 10089-10092. [CrossRef]

42. Case, D.A.; Aktulga, H.M.; Belfon, K.; Ben-Shalom, I.Y.; Brozell, S.R.; Cerutti, D.S.; Cheatham, T.E., III; Cisneros, G.A.; Cruzeiro, V.W.D.; Darden, T.A.; et al. Amber 1; University of California: San Francisco, CA, USA, 2018.

43. Case, D.A.; Aktulga, H.M.; Belfon, K.; Ben-Shalom, I.Y.; Brozell, S.R.; Cerutti, D.S.; Cheatham, T.E., III; Cisneros, G.A.; Cruzeiro, V.W.D.; Darden, T.A.; et al. Amber 20; University of California: San Francisco, CA, USA, 2020.

44. Glykos, N.M. Software news and updates carma: A molecular dynamics analysis program. J. Comput. Chem. 2006, 27, 1765-1768. [CrossRef] [PubMed]

45. Humphrey, W.; Dalke, A.; Schulten, K. VMD: Visual molecular dynamics. J. Mol. Graph. 1996, 14, 33-38. [CrossRef] 